\title{
The biological effect of human bone marrow derived mesenchymal stem cells expressing B7H4
}

\author{
Xueguang Zhang ${ }^{1}$, Xiying Luan ${ }^{2}$ \\ ${ }^{1}$ Stem Cell Research Key Laboratory of Jiangsu Province, Soochow University, Suzhou, China $;^{2}$ Department of Immunology, \\ Binzhou Medical School, Binzhou, China
}

The roles of $\mathrm{B} 7 \mathrm{H} 4$ expressed on human bone marrow derived mesenchymal stem cells (HBMSCs) mediating immunosuppression and their associated mechanisms were investigated in this study. HBMSCs from healthy donors were prepared by Ficoll-Plaque gradient centrifugation. Cells after 3 passages were used in the experiments. Flow cytometry analysis showed that HBMSCs highly expressed B7H4, a negative co-stimulatory molecule, but did not express the other molecules, such as CD80, CD86, HLA-DR, PDL1, CD40, CD40L and FasL, which were related to T cell activation. Furthermore, B7H4 was also detected on the HBMSCs by immunohistochemistry staining and RTPCR. Blocking B7H4 on HBMSCs by $\mathrm{B} 7 \mathrm{H4}$ monoclonal antibody resulted in restoring the $\mathrm{T}$ cell proliferation and attenuating the effects of HBMSCs on $T$ cell cycle through down-regulating the cell numbers in the G0/G1 phase or up-regulating the cell numbers in the $S$ phase. The results of confocal laser scanning microscopy observation showed that HBMSCs could inhibit NF-zB protein nuclear translocation in the activated $T$ cells. The effect was significantly weakened by blocking the B7H4 expression on the HBMSCs. The inhibition of HBMSCs on the T cell activation and proliferation required cell-to-cell contact, which were tested by transwell cell culture system. These results suggested that $\mathrm{B} 7 \mathrm{H} 4$ played an important role in regulating the $\mathrm{T}$ cell activation and proliferation, as well as the alteration of cell cycle and $\mathrm{NF}-\mathrm{zB}$ protein nuclear translocation in the activated $\mathbf{T}$ cells.

Keywords: B7H4, mesenchymal stem cells, bone marrow

Cell Research (2008) 18:s118. doi: 10.1038/cr.2008.208; published online 4 August 2008

$\overline{\text { Correspondence: Xueguang Zhang }}$

E-mail: smbxuegz@public1.sz.js.cn

Xueguang Zhang, MD/PhD, is the Professor of Immunology, Director of Medical Biotechnology Institute, and vice President of Soochow University. Dr Zhang received a PhD in biology from Montpelliar Scientific Technology University in France. Dr Zhang is an immunologist and his research has been focused on the regulatory mechanisms of costimulatory molecules in dendritic cell biology and tumor immunology. Dr Zhang is currently the Director of Key Laboratory in Stem Cell Research of Jiangsu Province. His research is also interested in the molecular mechanisms of costimulatory molecules involved in the regulatory roles of mesenchymal stem cells on immunocytes. 